\title{
Light Stimulates MSK1 Activation in the Suprachiasmatic Nucleus via a PACAP-ERK/MAP Kinase-Dependent Mechanism
}

\author{
Greg Q. Butcher, Boyoung Lee, Hai-Ying M. Cheng, and Karl Obrietan \\ Department of Neuroscience, Ohio State University, Columbus, Ohio 43210
}

\begin{abstract}
Signaling via the $42 / 44$ mitogen-activated protein kinase (MAPK) pathway has been shown to be a key intracellular signaling event that couples light to entrainment of the mammalian circadian clock located in the suprachiasmatic nucleus (SCN). Because many of the physiological effects of the MAPK pathway are mediated by extracellular signal-regulated kinase (ERK)-regulated kinases, it was of interest to identify kinase targets of ERK in the SCN. In this study, we examined whether mitogen- and stress-activated protein kinase 1 (MSK1) is a downstream target of ERK in the SCN and whether it couples to clock gene expression. Here we show that photic stimulation during the subjective night stimulates MSK1 phosphorylation at serine 360, an event required for robust kinase activation. Activated ERK and MSK1 were colocalized in SCN cell nuclei after photic stimulation. The in vivo administration of the MAP kinase kinase 1/2 inhibitor U0126 [1,4-diamino-2,3-dicyano-1,4-bis(o-aminophenylmercapto) butadiene] attenuated MSK1 phosphorylation. MSK1 phosphorylation was more responsive to late-night than early-night photic stimulation, indicating that MSK1 may differentially contribute to lightinduced phase advancing and phase delaying of the clock. The potential connection between pituitary adenylate cyclase-activating polypeptide (PACAP) (a regulator of clock entrainment) and MSK1 phosphorylation was examined. PACAP infusion stimulated MSK1 phosphorylation, whereas PACAP receptor antagonist infusion attenuated light-induced MSK1 phosphorylation in the SCN. In reporter gene assays, MSK1 was shown to couple to mPeriod1 via a cAMP response element-binding protein-dependent mechanism. Together, these data identify MSK1 as both a downstream target of the MAPK cascade within the SCN and a regulator of clock gene expression.
\end{abstract}

Key words: ERK; MAPK; p38; MSK1; CREB; period1; circadian; suprachiasmatic; PACAP

\section{Introduction}

In mammals, the dominant circadian pacemaker is located in the suprachiasmatic nucleus (SCN) of the hypothalamus. Its inherent timekeeping ability is derived from a discrete set of genes whose protein products form positive and negative limbs of a rhythmic transcriptional/translational feedback loop (for review, see Reppert and Weaver, 2001; Albrecht, 2002). Light is thought to entrain the clock by altering the expression of clock genes and, in turn, resetting the clock feedback loop (for review, see Meijer and Schwartz, 2003). For example, photic stimulation during the night triggers the expression of the core clock genes mPerl and mPer2 (Albrecht et al., 1997; Shigeyoshi et al., 1997), and the disruption of Per expression blocks the phase-shifting effects of light (Akiyama et al., 1999; Albrecht et al., 2001).

Photic information is relayed from the retina to the SCN via the retinohypothalamic tract (RHT), a branch of the optic nerve. The melanopsin expressing retinal ganglion cells that form the RHT secrete both glutamate and pituitary adenylate cyclase-

Received 0ct. 20, 2004; revised April 20, 2005; accepted April 20, 2005

This work was supported by National Institutes of Health Grants MH62335 and NS47176516161 (K.O.). G.Q.B. is a National Research Service Award trainee (Grant MH073374).

Correspondence should be addressed to Karl Obrietan, Department of Neuroscience, Ohio State University, Graves Hall, Room 4118, 333 West 10th Avenue, Columbus, OH 43210. E-mail: obrietan.1@osu.edu.

DOI:10.1523/JNEUROSCI.4361-04.2005

Copyright $\odot 2005$ Society for Neuroscience $\quad$ 0270-6474/05/255305-09\$15.00/0 activating polypeptide (PACAP) onto SCN cells (Hannibal et al., 2000, 2002; Gooley et al., 2001). The necessary role of glutamate as a signaling intermediate coupling light to clock entrainment has been well documented. For example, ionotropic glutamate receptor antagonist infusion into the SCN blocks light-induced phase shifts (Colwell et al., 1991). PACAP has been shown to be a potent and complex regulator of circadian clock entrainment (Hannibal et al., 1997; Harrington et al., 1999; Kawaguchi et al., 2003; Colwell et al., 2004). Interestingly, PACAP appears to function as an effector of multiple signaling pathways and augments the excitatory effects of glutamate on SCN cells (Chen et al., 1999; Harrington et al., 1999; Kopp et al., 2001; Dziema and Obrietan, 2002).

Although the precise set of cellular signaling events that couple glutamate and PACAP to clock entrainment has not been determined, both transmitters have been shown to stimulate activation of the p42/44 mitogen-activated protein kinase (MAPK) pathway (Barrie et al., 1997; Dziema and Obrietan, 2002). The MAPK pathway consists of three kinases: Raf, MAP kinase kinase (MEK), and extracellular signal-regulated kinase (ERK). During activation, ERK phosphorylates a number of cytosolic substrates and, depending on the strength of the stimulus, translocates to the nucleus and facilitates transcription activation (for review, see Cobb, 1999; Pearson et al., 2001; Roux and Blenis, 2004). In the SCN, light stimulates robust, phase-dependent, MAPK path- 
way activation (Obrietan et al., 1998; Butcher et al., 2003), and the disruption of light-induced MAPK activation blocks clock entrainment (Butcher et al., 2002).

The mechanism by which the MAPK cascade elicits transcription-dependent clock entrainment likely involves stimulation of a number of ERK-regulated kinases. Along these lines, we reported recently that light triggers activation of the ribosomal S6 family of kinases (RSKs) in the SCN (Butcher et al., 2004). RSKs function as central effectors of the MAPK cascade, stimulating transcription factor activation and altering chromatin structure. Additionally, another family of kinases, the mitogenand stress-activated protein kinases (MSKs), function as intermediates that couple the MAPK pathway to transcription activation (Deak et al., 1998; Arthur and Cohen, 2000; Wiggin et al., 2002; Arthur et al., 2004). MSKs have been shown to regulate gene transactivation via both transcription factor activation and histone phosphorylation (Wiggin et al., 2002; Soloaga et al., 2003). These observations raise the possibility that MSKs may couple the MAPK pathway to resetting of the circadian clock. Here we identify for the first time MSK1 as a light-responsive kinase that stimulates expression of the clock gene mPeriod1.

\section{Materials and Methods}

Light treatment. Initially, adult (6- to 14-week-old) C57BL/6 mice were entrained to a $12 \mathrm{~h}$ light/dark (LD) cycle for at least 2 weeks and then transferred to total darkness for two $24 \mathrm{~h}$ cycles. After dark adaptation, animals received a single light exposure (15 min, $100 \mathrm{lux}$ ) at one of three times: the middle of the subjective day [circadian time 6 (CT 6)], early night (CT 15), or late night (CT 22). CTs were approximated, based on Zeitgeber time (ZT), with ZT 0 denoting light on and ZT 12 denoting light off. Immediately after light treatment, animals were killed via cervical dislocation, and brains were removed under red light (Kodak series 2 filter $<10$ lux at cage level; Eastman Kodak, Rochester, NY). Brains were then placed in chilled, oxygenated physiological saline, cut into 500 $\mu \mathrm{m}$ coronal sections with an oscillating tissue slicer (OTS 2000; Electron Microscopy Sciences, Fort Washington, PA), and placed in 4\% paraformaldehyde (w/v in PBS) at room temperature for $4 \mathrm{~h}$. In total, it took $\sim 5$ min from decapitation to tissue fixation. In control experiments, we found that other, more time-consuming tissue-processing techniques, such as transcardial perfusion, resulted in a markedly attenuated kinase phosphorylation signal. This likely resulted from the transient nature of kinase phosphorylation. Tissue was then cryoprotected in 30\% sucrose (w/v) containing $2 \mathrm{~mm} \mathrm{Na}$ azide and $3 \mathrm{~mm} \mathrm{NaF}$ overnight at $4^{\circ} \mathrm{C}$. All procedures were in accordance with Ohio State University animal welfare guidelines.

Cannulation and infusion. Mice were cannulated in the lateral or third ventricles as described by Butcher et al. (2002) and allowed to recover for at least $10 \mathrm{~d}$ after cannulation. For the infusion, animals were restrained by hand under red light, and the infusate was delivered at a rate of 0.4 $\mu \mathrm{l} / \mathrm{min}$. To disrupt MAPKs, $3 \mu \mathrm{l}$ of 1,4-diamino-2,3-dicyano-1,4bis(o-aminophenylmercapto) butadiene (U0126) (10 mM), 4-[5-(4fluorophenyl)-2-[4-(methylsulfonyl)phenyl]- $1 H$-imidazol-4-yl]pyridine (SB203508) (10 mM), or a combination of the compounds was infused 45 min before photic stimulation. Control animals were infused with an equivalent volume of vehicle (DMSO). To disrupt PAC1 receptor activation, $5 \mu \mathrm{l}$ of PACAP 6-38 (0.5 mM) diluted in physiological saline was infused $15 \mathrm{~min}$ before photic stimulation. This concentration of PACAP 6-38 has been shown to significantly attenuate light-induced phase advances (Bergström et al., 2003). Two microliters of PACAP $(200 \mu \mathrm{M})$ were infused at CT 22; animals were killed 45 min later.

Immunohistochemistry. Brain sections $(500 \mu \mathrm{m})$ were thin cut $(40 \mu \mathrm{m})$ using a freezing microtome and placed in PBST (PBS with Triton X-100) with $2 \mathrm{~mm} \mathrm{Na}$ azide and $3 \mathrm{~mm} \mathrm{NaF}$. Endogenous peroxidases were quenched with $0.3 \% \mathrm{H}_{2} \mathrm{O}_{2}$ in PBST (15 min). Tissue was then blocked (1 h) in $5 \%$ goat serum/PBST and incubated (overnight, $4^{\circ} \mathrm{C}$ ) in rabbit anti-phospho-MSK1 (1:500 final dilution; Cell Signaling Technology, Beverly, MA), rabbit anti-phospho-ERK (1:2000; Cell Signaling Tech- nology), or rabbit anti-phospho-p38/MAPK (1:1000; Cell Signaling Technology). Next, sections were incubated $(2 \mathrm{~h})$ at room temperature in biotinylated anti-rabbit IgG (1:300; Vector Laboratories, Burlingame, $\mathrm{CA}$ ) and then placed in an avidin/biotin HRP complex for $1 \mathrm{~h}$ (prepared according to instructions of the manufacturer; Vector Laboratories). The signal was visualized by the addition of DAB-nickel-intensified substrate (Vector Laboratories) and mounted on gelatin-coated slides with Permount media (Fisher Scientific, Houston, TX).

Tissue used for fluorescent labeling was thin cut and blocked as described above and then incubated (overnight, $4^{\circ} \mathrm{C}$ ) in one or a combination of the following antibodies: rabbit polyclonal anti-phosphorylated ERK (1:1000; Cell Signaling Technology), mouse monoclonal neuronal nuclear-specific marker (NeuN) (1:1000; Chemicon, Temecula, CA), mouse monoclonal anti-phosphorylated ERK (1:5000; Sigma, St. Louis, MO), and rabbit polyclonal anti-phospho-MSK1 (1:250; Cell Signaling Technology). The following day, sections were incubated $(3 \mathrm{~h}$, room temperature) in Alexa Fluor-594-conjugated goat anti-rabbit IgG antibody (1:500; Molecular Probes, Eugene, OR) and/or Alexa Fluor-488conjugated goat anti-mouse IgG antibody (1:500; Molecular Probes). Sections were mounted on slides with Gelmount media (Biomedia, Foster City, CA). Brain sections were washed (five times, 5 min per wash) between each labeling step. Bright-field photomicrographs were captured using a 16 bit digital camera (Micromax YHS 1300; Princeton Instruments, Trenton, NJ) mounted on an inverted microscope (DM IRB; Leica, Nussloch, Germany). Fluorescent images were captured using a Zeiss (Oberkochen, Germany) 510 Meta confocal microscope (2$\mu \mathrm{m}$-thick optical section).

Reverse transcriptase-PCR. Animals were killed at CT 2, CT 8, CT 14, and CT 20, and brains were removed and cut into $500 \mu \mathrm{m}$ coronal sections as described above. Tissue was then frozen on slides with dry ice, and the SCNs were manually dissected and pooled from four animals for each time point. RNA was then isolated (RNA-Bee; Tel-Test, Friendswood, TX) and reverse transcribed using standard techniques. PCR was performed with primer sets against murine MSK1 (5'-cagttccatatgggagttgaccgt- $3^{\prime}$ and $5^{\prime}$-cttcatcattgcactcctggcaac- $\left.3^{\prime}\right)$ and cyclophilin $[\mathrm{CYC} \alpha$ (Uz and Manev, 1998)]. Reaction products were run on a $1 \%$ agarose gel containing ethidium bromide. Digital images were captured using an Eastman Kodak Image Station, and densitometric comparisons were performed to determine the ratio of CYC $\alpha$ to MSK1 for each time point.

Cell culture and stimulation. Cortical tissue was collected from embryonic (day 18) Sprague Dawley rat pups, washed in dissociation media (DM) [90 mм $\mathrm{Na}_{2} \mathrm{SO}_{4}, 30 \mathrm{~mm} \mathrm{~K}_{2} \mathrm{SO}_{4}, 16 \mathrm{~mm} \mathrm{MgCl}_{2}, 0.25 \mathrm{~mm} \mathrm{CaCl} 2,32$ mM HEPES, and $0.01 \%$ phenol red (Sigma), pH 7.7], and then incubated in digestion solution $[100 \mathrm{U} / \mathrm{ml}$ papain latex (Worthington, Freehold, $\mathrm{NJ}$ ) and $4.5 \mathrm{mg}$ cysteine (Sigma) in $\mathrm{DM}$ ] for $30 \mathrm{~min}$ at $37^{\circ} \mathrm{C}$. Tissue was then transferred to standard culture medium (Minimal Essential Medium; Invitrogen, Gaithersburg, MD) containing 1\% fetal bovine serum (Invitrogen), 2\% B27 (Invitrogen), and $100 \mathrm{U} / \mathrm{ml}$ penicillin/streptomycin (Invitrogen) and triturated into a single cell suspension. Cells were then plated onto poly-D-lysine-coated $9 \mathrm{~mm}^{2}$ glass coverslips at a density of 50,000 cells $/ \mathrm{cm}^{2}$. Media were changed $1 \mathrm{~h}$ after plating, and cells were maintained in a Napco (Winchester, VA) 6100 incubator $\left(37^{\circ} \mathrm{C}, 5.5 \%\right.$ $\mathrm{CO}_{2}$ ) for $10 \mathrm{~d}$. Media was replenished every third day.

Thirty minutes before experimental stimulation, tissue culture media were replaced with HEPES buffer (in mM: $137 \mathrm{NaCl}, 25$ glucose, 10 HEPES, $5 \mathrm{KCl}, 1 \mathrm{MgCl}_{2}$, and $3 \mathrm{CaCl}_{2}, \mathrm{pH} 7.4$ ) containing tetrodotoxin (1 $\mu \mathrm{M})$. Cells were then treated $(10 \mathrm{~min})$ with PACAP $(200 \mathrm{nM})$ or glutamate $(10 \mu \mathrm{M})$ and fixed (15 min) with paraformaldehyde (4\% w/v). Phosphorylated MSK1 (pMSK1) immunofluorescent labeling was performed as described above. To identify neurons, cells were also labeled with a microtubule-associated protein-2 (MAP2) monoclonal antibody (1:5000; Sigma). Cells were processed with Alexa Fluor-conjugated secondary antibodies, and digital images were captured as described above.

Transfection and luciferase assay. Initially, human embryonic kidney 293 (HEK 293) cells were seeded onto 24-well plates, grown to $90 \%$ confluency in DMEM containing 10\% fetal bovine serum and $1 \%$ penicillin, and then transfected using Lipofectamine 2000 (Invitrogen). A total volume of $1.0 \mu \mathrm{g}$ of DNA was transfected per well. The following 
plasmids were used: constitutively active MSK1 [CA-MSK1 (Frodin et al., 2000)], dominant-negative MSK1 [DN-MSK1 (Arthur et al., 2004)], dominant-negative cAMP response element-binding protein (CREB) [A-CREB (Ahn et al., 1998)], and a murine period 1 (mPerl) reporter construct. The mPer 1 construct consisted of a $2.2 \mathrm{~kb}$ fragment of the $5^{\prime}$ regulatory region of the second of the two mPer1 promoters [exon $1 \mathrm{~B}$ promoter (Yamaguchi et al., 2000)] cloned into the pGL3-enhancer vector (Promega, Madison, WI). This reporter construct is analogous to the reporter used by Gekakis et al. (1998). Cells were serum starved $16 \mathrm{~h}$ before stimulation $(8 \mathrm{~h})$ with 12 -O-tetradecanoylphorbol-13-acetate (TPA; $200 \mathrm{ng} / \mathrm{ml}$ ). Cells were lysed $48 \mathrm{~h}$ after transfection, and luciferase reporter expression was determined using the method described by Ford and Leach (1998). To verify the efficacy of our transfection approach, HEK 293 cells were grown to confluency on $9 \mathrm{~mm}^{2}$ coverslips and cotransfected with a cytomegalovirus-green fluorescent protein (GFP) reporter construct and FLAG-tagged DN-MSK1. Forty-eight hours later, cells were fixed and blocked as described above and labeled using a polyclonal antibody directed against GFP (1:1000; Molecular Probes) and a monoclonal anti-FLAG antibody (1:2500; Sigma) at $4^{\circ} \mathrm{C}$ overnight. Alexa-conjugated secondary antibodies were used (as described above) to visualize the signal. Cells were also labeled with Hoechst 33258 (1 $\mu \mathrm{g} / \mathrm{ml}$; Molecular Probes) and then mounted with Gelmount.

Data analysis. All data were quantified using MetaMorph software (Universal Imaging Corporation, West Chester, PA). For cell counts from DAB-labeled tissue, an intensity threshold filter was initially applied to the image. The filter eliminated nonspecific background labeling from analysis. Digital circles were then overlaid on regions with a detectable signal above threshold, now defined as positive cells, and counted. Cell counts were averaged over three consecutive central SCN sections for each experimental animal. For quantitation of pMSK1 expression in cultured neurons, digital ovals were placed over nuclei delineated by MAP2 labeling, and signal intensity values were collected from each of these regions. Cells were counted as PMSK1 positive if the average intensity level within a given nuclear region was $\geq 75 \%$ of the mean maximal signal found across all conditions. The fluorescent images from a minimum of four coverslips (four regions per coverslip) were captured. Significance was determined using the two-tailed Student's $t$ test and was defined as $p<0.05$. All data are expressed as the mean \pm SEM.

Materials. PACAP and PACAP 6-38 were obtained from Bachem (Bubendorf, Switzerland). U0126 and SB203580 were purchased from Calbiochem (La Jolla, CA). Luciferin potassium salt was acquired from Promega. All other reagents were obtained from Sigma unless otherwise indicated.

\section{Results}

\section{Light-induced MSK1 activation}

As an initial step in our examination of MSK1 in the SCN, C57BL/6 mice were entrained to a $12 \mathrm{~h} \mathrm{LD} \mathrm{cycle} \mathrm{and} \mathrm{then} \mathrm{dark}$ adapted for $2 \mathrm{~d}$. At the end of this period, mice were exposed to light (15 min, 100 lux) during either subjective day (CT 6) or subject night (CT 15 and CT 22) time points. Immediately after photic stimulation, animals were killed, and SCN-containing brain sections were immunohistochemically processed for the serine 360 phosphorylated form of MSK1. We chose to use an antibody that detects the phosphorylation state of serine 360 for two reasons. First, because serine 360 is flanked by prolines in the -2 and +1 positions, it is a likely target for both ERK and p38/ MAPK (for review, see Roux and Blenis, 2004; Smith et al., 2004). Second, serine 360 phosphorylation is required to stimulate full MSK1 activity (McCoy et al., 2005). Thus, by monitoring phosphorylation at serine 360 , it is possible to infer the activation state of the kinase. Photic stimulation during both the early (CT 15) and late (CT 22) subjective night produced a significant increase in the number of cells expressing pMSK1 (Fig. $1 A, B$ ). In contrast, photic stimulation during the middle of the subjective day (CT 6) failed to activate MSK1. Light-induced MSK1 phosphorylation was highest in the ventral SCN, corresponding to the region that
A
A
듬
$\frac{5}{2}$

CT 6
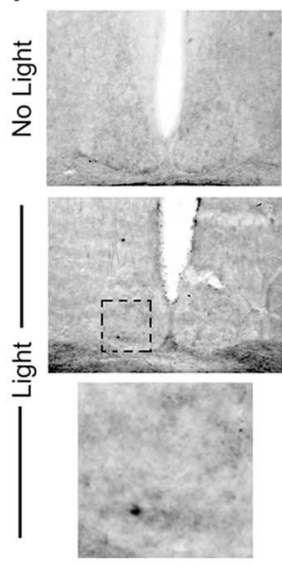

B

B
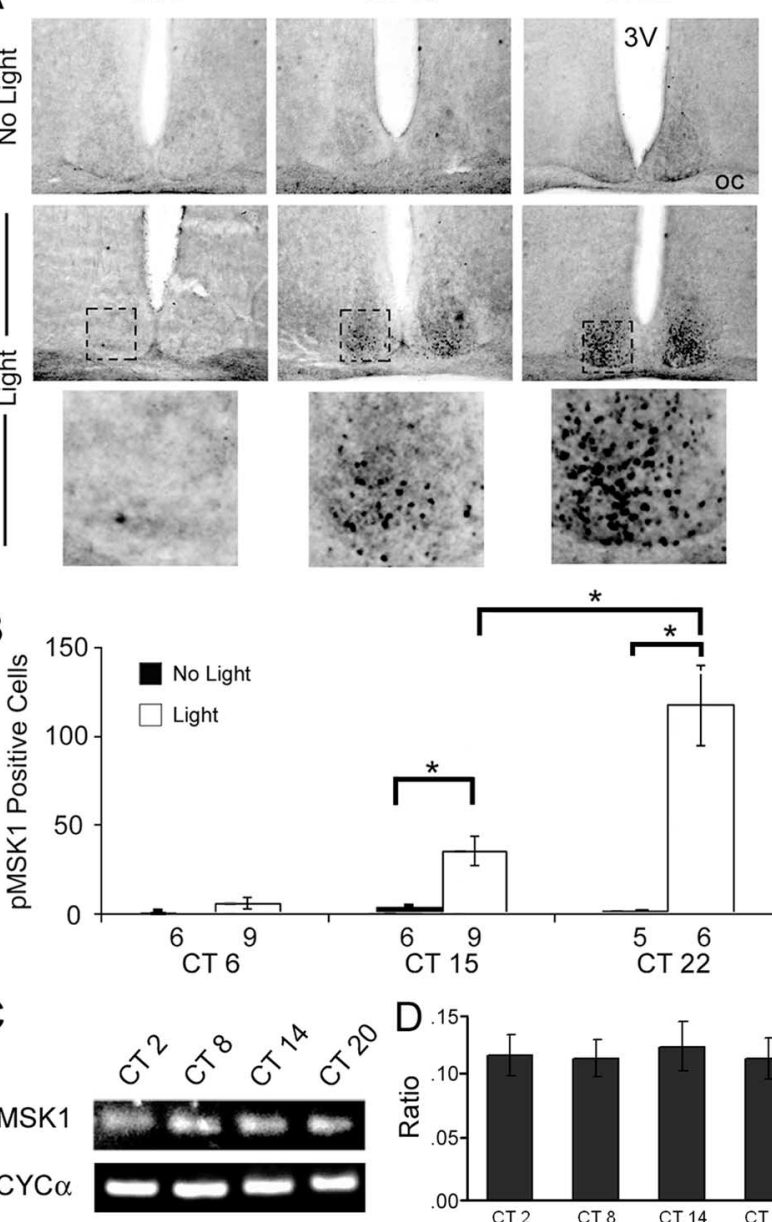

$\Gamma$
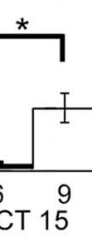

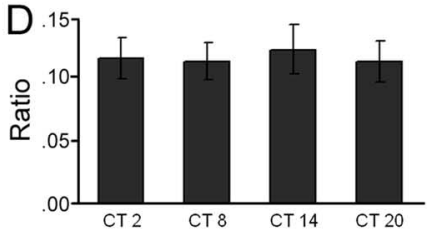

Figure 1. Light induces MSK1 phosphorylation. $\boldsymbol{A}$, Mice were exposed to light during the subjective day (CT 6) or subjective night (CT 15 and CT 22). Representative images of SCNcontaining coronal brain sections immunohistochemically labeled for pMSK1 are shown. Boxed regions are enlarged below each respective image. Box, $100 \mu \mathrm{m}^{2}$. 3V, Third ventricle; $0 \mathrm{C}$, optic chiasm. $\boldsymbol{B}$, Mean number of pMSK1-positive cells per SCN section. Light exposure during the subjective day (CT 6) did not elicit pMSK1 ( $p>0.1$ ). Photic treatment at either night time point (CT 15 or CT 22) produced a significant increase in the number of cells containing PMSK1 relative to control animals killed at the same time points. Light treatment at CT 22 was also found to produce a significant increase in the number of pMSK1-positive cells compared with light exposure at $\mathrm{CT} 15 .{ }^{*} p<0.05$. Numbers below each bar represent the number of animals used for each condition. C, RT-PCR analysis of MSK1 and CYC $\alpha$ mRNA expression in the SCN. D, Densitometric analysis of data presented in $C$ revealed no significant variation in total MSK1 mRNA expression as a function of circadian time. Results are presented as the ratio of the MSK1 to cyclophilin. Data were averaged from triplicate determinations for each time point.

receives the greatest degree of innervation from the RHT (for review, see Moore, 1996; Moore and Silver, 1998). Interestingly, pMSK1 induction was more responsive (twofold to threefold) to late-night than to early-night light pulses. For example, light exposure at CT 22 stimulated pMSK1 expression in $117.0 \pm 22.6$ cells per SCN section, whereas at CT 15 light stimulated pMSK1 expression in $35.6 \pm 8.3$ cells per SCN section (Fig. $1 B$ ). In the absence of photic stimulation, we did not observe significant variations in pMSK1 expression over the three time points examined, suggesting that pMSK1 is not regulated by the clock timing mechanism. Reverse-transcriptase (RT)-PCR was used to examine MSK1 mRNA expression as a function of circadian time (Fig. 1C). SCN tissue was collected at CT 2, CT 8, CT 14, and CT 20, and the ratio of MSK1 mRNA levels to cyclophilin [a nonrhythmically expressed gene (Uz and Manev, 1998)] mRNA levels was 


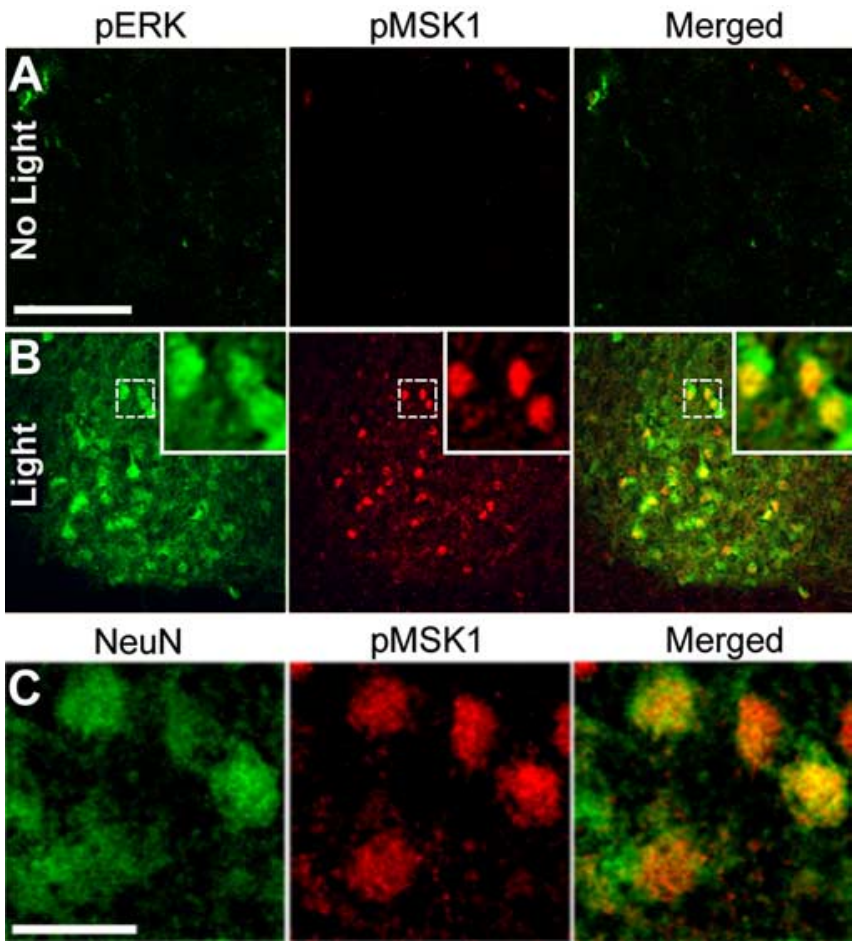

Figure 2. Light triggers the colocalized expression of pMSK1 and pERK in neuronal nuclei. Compared with controls (No Light, $\boldsymbol{A}$ ), photic stimulation (Light, $\boldsymbol{B}$ ) elicited an increase in the number of pMSK1-positive and pERK-positive cells. Merging of the pERK and pMSK1 signals revealed that the expression of the activated forms of the two kinases was colocalized. Scale bar, $100 \mu \mathrm{m}$. The boxed region is magnified in the inset. C, A representative image of ventral SCN tissue from a light-treated animal labeled with antibodies directed against pMSK1 (red) and NeuN (green). The colocalized expression of pMSK1 and NeuN indicates that activated MSK1 is expressed in neuronal nuclei. Scale bar, $15 \mu \mathrm{m}$.

determined (Fig. 1D). Relative levels of MSK1 mRNA did not significantly vary over the four time points examined, indicating that MSK1 is not rhythmically regulated. Collectively, these data reveal that light-induced MSK1 phosphorylation is phase restricted to the night and that phase-advancing and phasedelaying light pulses differentially regulate its activation.

Given that MSK1 is a potential target of ERK and that light stimulates ERK activation in the SCN, we examined whether light triggers the colocalized expression of phosphorylated forms of the two kinases. To this end, coronal tissue sections from animals exposed to light (CT 22, $15 \mathrm{~min}, 100 \mathrm{lux}$ ) and control (no light) animals were double immunolabeled for pMSK1 and the activated (Thr 202/Tyr 204 phosphorylated) forms of ERK-1 and ERK-2 (pERK). Compared with controls, light stimulated robust pERK and pMSK1 expression in the ventrolateral SCN (Fig. $2 A, B)$. Merging of the images revealed that the majority of pMSK1-positive cells also express activated ERK (indicated by the yellow hue). pERK is expressed in both the cytoplasm and nucleus, whereas MSK1 is exclusively nuclear (Deak et al., 1998). The magnification insets in Figure $2 B$ revealed this general staining pattern for the two kinases. High-magnification microscopy of SCN tissue (light exposure, CT 22) for pMSK1 and the neuronal-specific nuclear marker NeuN confirmed the nuclear localization of pMSK1 (Fig. 2C).

\section{Kinase coupling to MSK1}

Both ERK and p38/MAPK are expressed in the SCN (Obrietan et al., 1998; Pizzio et al., 2003) and capable of phosphorylating MSK1 at serine 360. To determine which of these pathways cou- ples light to serine 360 phosphorylation, we used a ventricular infusion approach to block MEK (and thus ERK) and p38/MAPK enzymatic activity. To this end, animals were infused with drug vehicle (DMSO), the MEK1/2-specific inhibitor U0126 (10 mM), the p38/MAPK-specific inhibitor SB203508 (10 mM), or a combination of the two inhibitors. Compared with vehicle-infused, light-stimulated animals, U0126 significantly reduced the number of pMSK1-expressing cells (Fig. $3 A, B,{ }^{*} p<0.001$ ), indicating that the MAPK pathway couples light to MSK1 phosphorylation. Infusion of SB203508 also attenuated MSK1 activation; however, the level of inhibition failed to reach significance relative to the DMSO-infused light-treatment condition $(p>0.06)$. It should be noted that DMSO elicited a modest, nonsignificant $(p>0.25)$ reduction in the number of pMSK1-positive cells (compare Figs. $1 B, 3 B)$. The infusion of both $\mathrm{U} 0126$ and SB203508 reduced light-induced MSK1 phosphorylation to the level of control animals. These data indicate that the MAPK cascade is the dominant regulator of MSK1 activation in the SCN. The inhibitory effects of SB203508 also suggest a role for the p38/MAPK pathway in coupling light to MSK1 activation. However, a cautionary note was raised when we were not able to detect significant light-induced p38/MAPK activation in the SCN (data not shown).

\section{PACAP-dependent MSK1 activation}

The observation that MSK1 was differentially regulated by earlyand late-night light pulses suggests that different upstream regulatory events are actuated by light at these two time points. Within this context, it is of interest to note that PACAP has been shown to have complex early-night- and late-night-specific effects on clock entrainment (Chen et al., 1999; Hannibal et al., 2001; Kawaguchi et al., 2003) and can stimulate clock gene expression (Nielsen et al., 2001; Minami et al., 2002). The phasespecific effects of both PACAP and MSK1 raised the possibility that PACAP and MSK1 form a signaling cassette. To begin to address this issue, we examined whether PACAP stimulates MSK1 phosphorylation. To this end, mice were infused with $\operatorname{PACAP}(200 \mu \mathrm{M})$ at CT 22 . Forty-five minutes later, animals were killed and the tissue was processed for PMSK1 expression. Relative to vehicle-infused animals, PACAP elicited a marked increase in pMSK1 expression within the SCN (Fig. 4A). Consistent with the expression pattern of PACAP receptors (Kalamatianos et al., 2004), PACAP was also found to elicit pMSK1 expression in periventricular regions dorsal to the SCN. Discernable PACAPinduced pMSK1 expression was observed in $75 \%$ of the animals $(n=8)$. Vehicle infusion did not alter MSK1 expression $(n=5)$ relative to control, no light conditions (Fig. 1A). As expected, PACAP infusion also stimulated ERK phosphorylation in the SCN (Fig. 4B). To quantitate MSK activation, rat primary neuronal cultures were stimulated with PACAP (200 nM) or glutamate $(10 \mu \mathrm{M})$ and then immunolabeled for pMSK1. Relative to control conditions (mock stimulation), both PACAP and glutamate elicited a significant increase in the number of neurons expressing pMSK1 (Fig. $4 C, D,{ }^{\star} p<0.05$ ). Interestingly, PACAP was a much more effective regulator of pMSK1 than glutamate; PACAP triggered pMSK1 expression in approximately twice as many neurons as glutamate. Similar results were observed using mouse neuronal cultures (data not shown).

To assess whether PACAP receptor activation regulates lightinduced pMSK1 in the SCN, mice were infused with PACAP 6-38. This truncated form of PACAP functions as a potent antagonist of the PAC1 receptor (Robberecht et al., 1992) and thus should block the physiological effects of PACAP release from the RHT. Initially, mice were infused with either vehicle (saline) or 


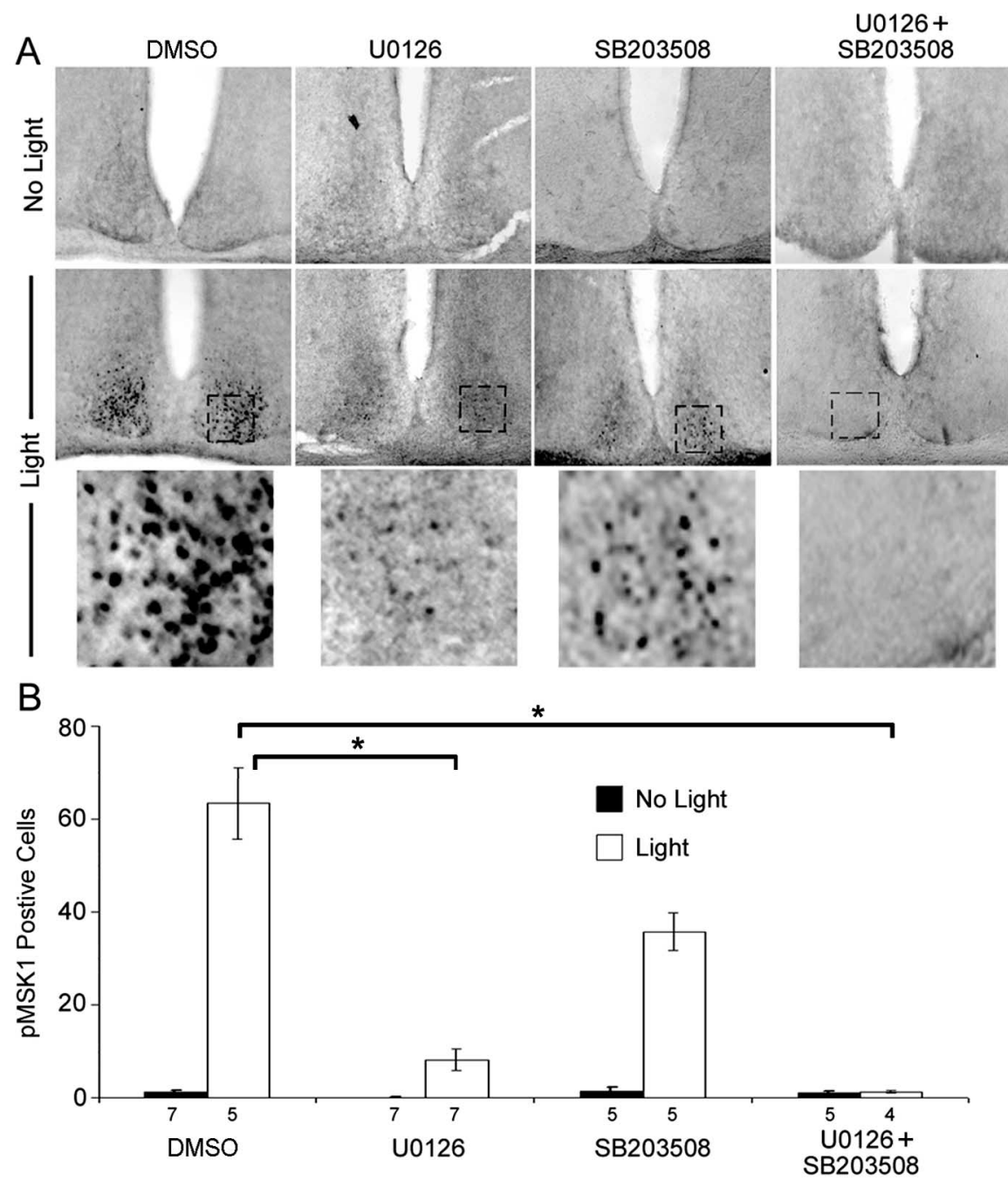

Figure 3. The MAPK and p38/MAPK pathways couple light to MSK1 activation. Cannulated animals were infused with $3 \mu$ l of DMSO (vehicle), U0126, SB203508, or a combination of U0126 and SB203508 45 min before light exposure (15 min, 100 lux) at CT 22. A, Representative coronal tissue sections immunolabeled for phosphorylated MSK1. Boxed regions are magnified below each image. $\boldsymbol{B}$, Average number of pMSK1-positive cells per SCN. Disruption of the MAPK pathway significantly reduced the number of positive cells relative to DMSO infusion. SB203508 attenuated light-induced MSK1 activation; however, this effect failed to reach significance relative to DMSO infused, light-treated animals ( $p>0.06$ ). The combined administration of U0126 and SB203508 blocked MSK1 phosphorylation. ${ }^{*} p<0.001$, relative to the DMS0-infused light-treated condition. Numbers below each bar represent the number of animals used for each condition.

PACAP 6-38 15 min before photic stimulation at CT 15 or CT 22. Relative to control conditions (vehicle infusion with photic stimulation), the infusion of PACAP 6-38 significantly $\left({ }^{*} p<0.001\right.$ ) attenuated the capacity of light to stimulate pMSK1 expression (Fig. $5 A, B$ ). In the absence of light, PACAP 6-38 did not alter the number of pMSK1-positive cells. These data suggest that PACAP signaling through the PAC1 receptor couples light to MSK1 activation.

\section{MSK1 stimulates mPer1}

As noted in Introduction, much of the transcriptional potential of the MAPK cascade is exerted by ERK-regulated kinases. As a potent regulator of CREB phosphorylation and histone modification, MSK1 is well positioned to couple light to immediate early clock gene expression.

To examine the role of MSK1 as a regulator of clock gene expression, HEK 293 cells were transfected with an mPer1luciferase reporter construct and DN-MSK1 (Arthur et al., 2004) or CA-MSK1 (Frodin et al., 2000). TPA $(200 \mathrm{ng} / \mathrm{ml})$ induced a marked increase in mPerl expression that was blocked by cotransfection with DN-MSK1 (Fig. 6A,B). Pretreatment with U0126 $(10 \mu \mathrm{M})$ also blocked TPA-induced mPer1 expression, indicating that TPA couples to mPer 1 via a MAPK/MSK1-dependent mechanism. To further examine the role of MSK1 as a regulator of mPer 1 expression, cells were cotransfected with mPerl-luciferase and CA-MSK. Compared with an mPer1-luc/ empty vector (pcDNA3.1) transfection, CA-MSK1 triggered a significant sixfold increase in mPer1-dependent transcription (Fig. 6A). Finally, to determine whether MSK1 couples to mPer1 via a CREB-dependent mechanism, cells were also transfected with A-CREB, a dominant-negative regulator of CREBmediated transcription (Ahn et al., 1998). A-CREB attenuated the capacity of MSK to stimulate mPer1-dependent transcription (Fig. 6A). Together, these data suggest that a PACAP/MAPK/MSK1/CREB signaling cassette couples photic information to period 1 expression.

\section{Discussion}

The goal of this study was to begin a systematic characterization of the ERKregulated kinase MSK1 in the SCN. Here we report that photic stimulation triggered the phase-dependent phosphorylation of MSK1 at serine 360. Stimulation was maximal during the late subjective night, and signaling via the $\mathrm{PAC1}$ receptor appeared to be central to MSK1 activation. As a regulator of period 1 expression, MSK1 may be a key intermediate in the set of signaling events that couples light to clock entrainment.

Light entrainment of the circadian clock appears to be a transcriptionally dependent process. Correlative support for this concept has been gathered from studies showing that light-induced gene expression precedes clock entrainment (Kornhauser et al., 1990; Rusak et al., 1990; Albrecht et al., 1997; Shigeyoshi et al., 1997; Best et al., 1999; Bae and Weaver, 2003). Causal evidence comes from studies showing that the disruption of clock gene expression disrupts the phaseshifting effects of light and glutamate (Akiyama et al., 1999; Albrecht et al., 2001; Wakamatsu et al., 2001; Trischkau et al., 2003).

One cellular signaling pathway that has been shown to couple photic information to clock entrainment is the MAPK pathway. We and several other groups have demonstrated that lightinduced MAPK pathway activation is phase restricted to the nighttime portion of the $24 \mathrm{~h}$ cycle (Obrietan et al., 1998; Butcher et al., 2003; Coogan and Piggins, 2003). Furthermore, disruption of signaling via the MAPK cascade blocks both light-induced gene expression (Dziema et al., 2003) and light entrainment of the circadian clock (Butcher et al., 2002; Coogan and Piggins, 2003; Cheng et al., 2004). Interestingly, dysfunctional SCN pace- 
maker activity removes the phaserestricted responsiveness of the MAPK pathway to light (Hughes et al., 2004). Together, these data indicate that the MAPK pathway is a key signaling intermediate in light entrainment of the clock and that the clock imposes phase dependency over light responsiveness of the MAPK pathway.

Within the context of gene regulation, two families of kinases, RSKs and MSKs, appear to be central effectors of the MAPK cascade. In a previous study, we found that both light and the endogenous clock timing mechanism regulate the activation state of RSK1 (Butcher et al., 2004). MSK1 is related to the RSK family of kinases (Deak et al., 1998). It is a serine/threonine kinase that is composed of two distinct domains: an N-terminal kinase that phosphorylates MSK1 substrates, and a C-terminal kinase that functions in an autoregulatory role (Deak et al., 1998; Smith et al., 2004; McCoy et al., 2005). MSK1 activation is mediated in part by a series of phosphorylation steps within an approximate 50 amino acid residue linker region that connects the $\mathrm{C}$ - and $\mathrm{N}$-terminal kinases. Although these steps are analogous to those that drive RSK activation, there are distinct differences between the two kinases. Principal among them is that MSK1 activation is not dependent on PDK1 (phosphoinositide-dependent protein kinase-1) phosphorylation within the $\mathrm{N}$ terminus (Williams et al., 2000). Furthermore, unlike RSKs, MSK1 is exclusively localized to the nucleus, and its activation is regulated by both the MAPK pathway and the p38/MAPK pathway (Deak et al., 1998; Pierrat et al., 1998). Interestingly, MSK1 has been shown to be a much more effective CREB kinase than RSK2 (Deak et al., 1998; Pierrat et al., 1998; Arthur and Cohen, 2000).

Initially, we found that light exposure during the night triggered a marked increase in the number of cells expressing the serine 360 phosphorylated form of MSK1 in the SCN. Under control (no light) conditions, there was a near total absence of pMSK1 expression in the SCN. Furthermore, pMSK1 expression was not detectable within the surrounding hypothalamic and thalamic brain regions or within the forebrain (data not shown). This absence of pMSK1 expression is surprising given that activated ERK is found in a variety of brain regions under control conditions (Obrietan et al., 1998). The lack of pMSK1 expression may reflect the limited sensitivity of the staining technique used. Conversely, the robust light-specific activation of pMSK1 in the SCN may indicate that MSK1 is primed to convey photic information. It should also be noted that the lack of basal pMSK1 expression in the SCN is in contrast to data showing that pRSK1 and pERK expression are rhythmically regulated (Obrietan et al., 1998; Butcher et al., 2004). This divergence in ERK/RSK1 and cells examined for each condition.
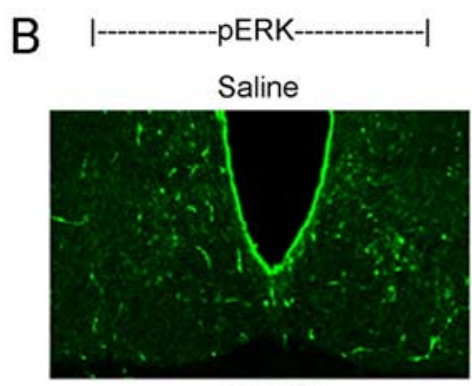

PACAP

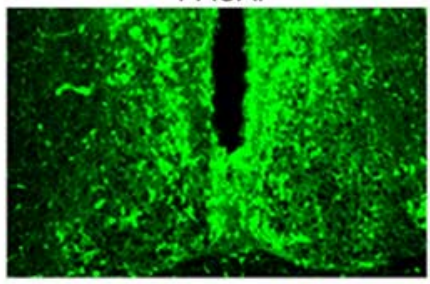

D

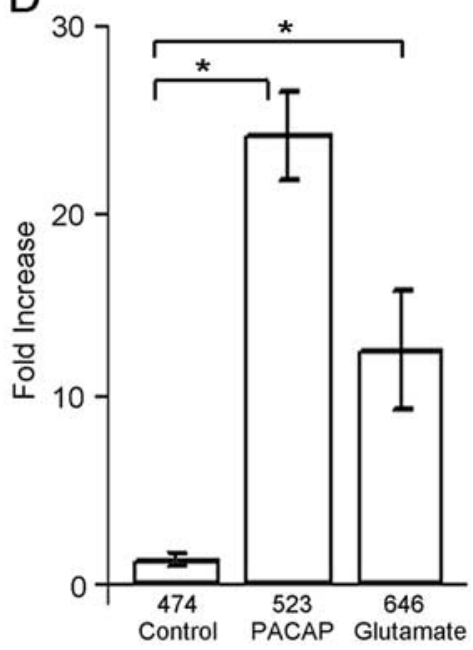

Figure 4. PACAP stimulates MSK1 phosphorylation. $\boldsymbol{A}$, Representative pMSK1 immunostaining from saline (vehicle)- and PACAP (200 $\mu \mathrm{m}$ )-infused mice. Mice were infused via the lateral ventricle at CT 22 and killed 45 min later. Relative to vehicle infusion, PACAP elicited a marked increase in pMSK1 expression in the periventricular regions, including the SCN. $\boldsymbol{B}$, PACAP also

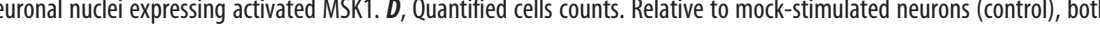
stimulation relative to control levels of pMSK, which were normalized to a value of 1 . Numbers below bars indicate the number of

MSK1 activity would likely contribute to the distinct gene expression patterns occurring under control conditions and after photic stimulation.

In this study, we used several approaches to determine whether the MAPK pathway regulates PMSK1 expression. Initially, immunofluorescence-based double labeling was used to examine the expression pattern of the activated forms of the kinases after light exposure. In the majority of SCN cells, we observed a spatial and temporal colocalization of the two kinases within cellular nuclei. Given that the inactive form of ERK is anchored to MEK within the cytoplasm (Fukada et al., 1997), these data indicate that light triggers the translocation of ERK. Indeed, we have shown previously that photic stimulation triggers the nuclear accumulation of activated ERK (Butcher et al., 2003). To directly test whether ERK stimulates MSK1 phosphor- 


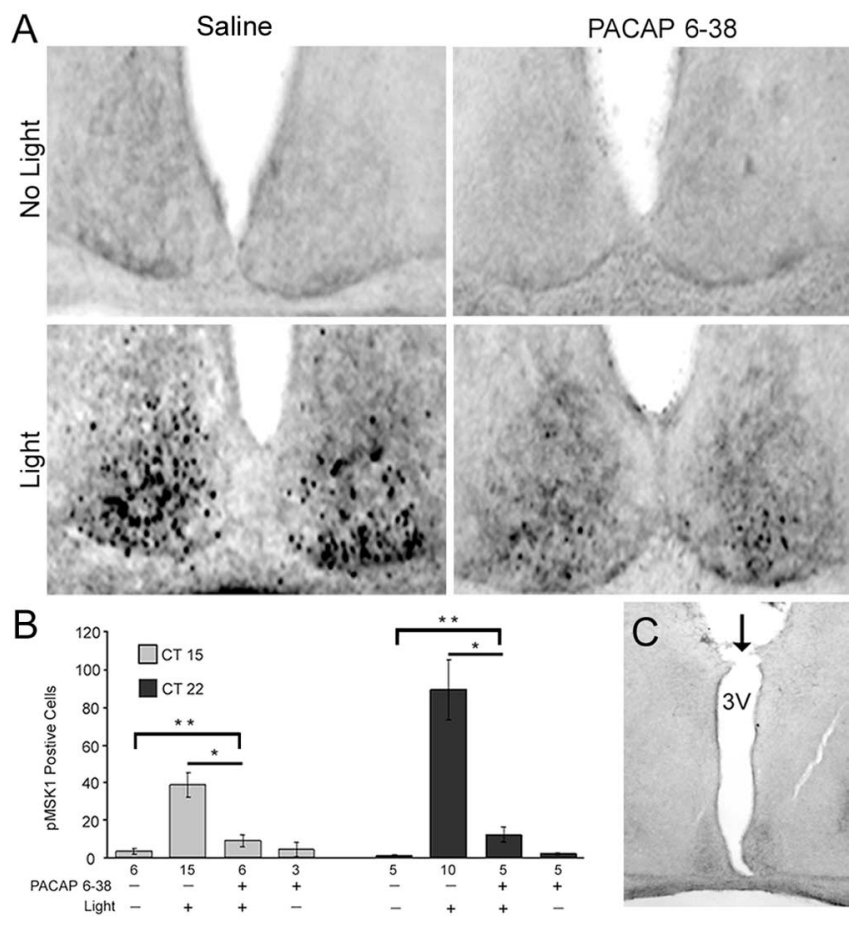

Figure 5. Inhibition of the PAC1 receptor significantly attenuates light-induced MSK1 phosphorylation. Animals were infused with the PAC1-specific antagonist PACAP 6-38 or saline (vehicle) $15 \mathrm{~min}$ before a light treatment ( $15 \mathrm{~min}, 100 \mathrm{lux}$ ) at $\mathrm{CT} 15$ and CT 22.A, Representative pMSK1 immunolabeled sections from each of the four treatment groups at CT 22. $\boldsymbol{B}$, Compared with saline infusion, the infusion of PACAP 6-38 significantly reduced the capacity of light to elicit pMSK1 expression ( ${ }^{*} p<0.001$ ). Although PACAP 6-38 attenuated the number of pMSKexpressing cells, a modest but significant residual pMSK1 signal was observed compared with controls $\left({ }^{* *} p<0.05\right)$. Numbers below bars denote the number of animals used for that condition. C, Histological examination of cannula placement within the dorsal third ventricle (3V). Black arrow denotes the location of the cannula tip.

ylation at serine 360 , we infused the MEK1/2 inhibitor U0126 into the third ventricle. This technique relies on the diffusion of U0126 into the periventricular region containing the SCN. In control experiments, we have shown that U0126 diffuses into the SCN and effectively blocks ERK activation (Butcher et al., 2002, 2004; Dziema et al., 2003). The data presented here reveal that the disruption of MAPK signaling significantly attenuates lightinduced MSK1 phosphorylation, thus identifying MSK1 as an ERK-regulated kinase in the SCN.

As noted above, MSK1 can also be activated by the p38/MAPK pathway. Because infusion of U0126 did not totally suppress MSK1 phosphorylation, we tested the potential contribution of the p38/MAPK pathway. Infusion of the p38/MAPK inhibitor SB203508 elicited a modest reduction in the number of pMSK1positive cells after a light pulse. Given the relative specificity of SB203508 (Davies et al., 2000) for neuronally enriched p38/ MAPK isoforms (Wang et al., 1997; Jiang et al., 1998), these results suggest that $\mathrm{p} 38$ /MAPK may play an ancillary role in MSK1 phosphorylation. Interestingly, the combined infusion of U0126 and SB203508 totally blocked light-induced pMSK1 expression. This result lends additional support to the idea that both kinase pathways contribute to light-induced MSK1 phosphorylation in the SCN. As noted in Results, we were not able to conclusively show that $\mathrm{p} 38$ /MAPK was activated by light in the SCN. This is in contrast to work by Pizzio et al. (2003) who reported that p38/ MAPK is light responsive. This discrepancy is the likely result of different methodological approaches used by the two groups.
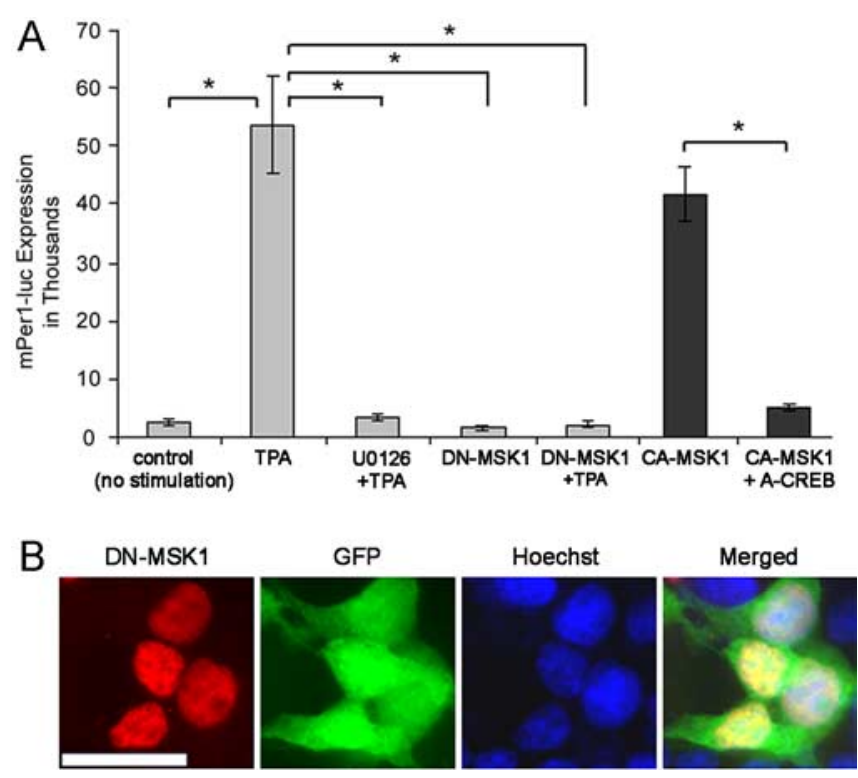

Figure 6. MSK1 stimulates mPer1-dependent transcription. $\boldsymbol{A}$, HEK 293 cells were transfected with an mPer1-luciferase reporter construct ( $200 \mathrm{ng} /$ well) and one or a combination of the following constructs: DN-MSK1 (800 ng/well), CA-MSK1 (200 ng/well), or A-CREB (800 $\mathrm{ng} /$ well). pCDNA3.1 (empty vector) was cotransfected as needed to bring the total to $1 \mu \mathrm{g} / \mathrm{well}$. Relative to mock-stimulated cells, TPA (200 ng/ml) elicited a marked increase in mPer1luciferase expression. In contrast, TPA-induced mPer1 expression was attenuated by cotransfection with DN-MSK1 and by pretreatment (30 min) with U0126 (10 $\mu \mathrm{M})$. CA-MSK1 (black bars) stimulated robust mPer1-luciferase expression. Cotransfection with A-CREB significantly attenuated the effects of CA-MSK1, indicating that MSK couples to mPer1-dependent transcription via a CREB-dependent mechanism. Data are expressed as absolute intensity units. Experiments were performed four times, and representative data were averaged from quadruplicate determinations. ${ }^{*} p<0.001$. B , HEK 293 cells were immunolabeled for FLAG-tagged DN-MSK1 (red) and the cotransfection marker protein GFP (green) and stained with Hoechst 33258 (blue). Note the nuclear-specific expression of DN-MSK1. Scale bar, $25 \mu \mathrm{m}$.

One striking finding of this study was that MSK1 was much more responsive to late-night than early-night photic stimulation. Light exposure during the late night triggered MSK1 activation in two to three times as many SCN cells as light exposure during the early night. This difference does not appear to be the result of circadian variations in MSK1 expression; levels of the kinase transcript were approximately equivalent across a $24 \mathrm{~h}$ period. One potential explanation for this disparate sensitivity is that different upstream cellular signaling events are stimulated by light during the early and late night. Interestingly, several studies have shown that the phase-advancing effects of light are more severely disrupted than the phase-delaying effects of light in PACAP- and PAC1-deficient mice (Hannibal et al., 2001; Kawaguchi et al., 2003). The parallels in the late-night effects of PACAP and the late-night responsiveness of MSK1, coupled with the finding that PACAP is a potent activator of the MAPK pathway in SCN neurons (Dziema and Obrietan, 2002), raised the possibility that PACAP couples photic information to MSK1.

To address whether PACAP and MSK1 form a functional signaling cassette in vivo, we infused the $\mathrm{PAC} 1$ receptor antagonist PACAP 6-38 into the third ventricle. Infusion of this peptide has been shown to attenuate the phase-shifting effects of light (Bergström et al., 2003). Pretreatment with PACAP 6-38 significantly reduced the number of pMSK1-positive cells after light treatment, indicating that PACAP potently regulates MSK activation in the SCN. In addition, infusion of PACAP stimulated MSK1 phosphorylation. Together, these data raise the possibility that a 
late-night signaling cassette formed between PACAP and MSK1 contributes to the phase-advancing effects of light.

The simplest route by which MSK1 could regulate clock phase would be through activation of clock gene expression. Interestingly, MSK has been shown to regulate CREB phosphorylation at serine 133 (Deak et al., 1998; Pierrat et al., 1998; Arthur and Cohen, 2000; Arthur et al., 2004), an event necessary for CREmediated gene expression (Gonzalez and Montminy, 1989). Furthermore, the 5 ' regulatory region of murine period 1 has multiple CRE sites that have been proposed to play a central role in its activity-dependent expression (Yamaguchi et al., 2000; Travnickova-Bendova et al., 2002; Trischkau et al., 2003). In our reporter gene studies, we found that MSK1 coupled the MAPK cascade to mPerl via a CREB-dependent mechanism. These data fit nicely with previous studies showing that both MAPK signaling and CREB facilitate period1 expression (Cermakian et al., 2002; Travnickova-Bendova et al., 2002). Collectively, these studies identify a light-activated signaling pathway that may directly impinge on the core clock timing mechanism.

\section{References}

Ahn S, Olive M, Aggarwal S, Krylov D, Ginty DD, Vinson C (1998) A dominant-negative inhibitor of CREB reveals that it is a general mediator of stimulus-dependent transcription of c-fos. Mol Cell Biol 18:967-977.

Akiyama M, Kouzu Y, Takahashi S, Wakamatsu H, Moriya T, Maetani M, Watanabe S, Tei H, Sakaki Y, Shibata S (1999) Inhibition of light- or glutamate-induced mPerl expression represses the phase shifts into the mouse circadian locomotor and suprachiasmatic firing rhythms. J Neurosci 19:1115-1121.

Albrecht U (2002) Functional genomics of sleep and circadian rhythm. Invited review: regulation of mammalian circadian clock genes. J Appl Physiol 92:1348-1355.

Albrecht U, Sun ZS, Eichele G, Lee CC (1997) A differential response of two putative mammalian circadian regulators, mper 1 and mper2, to light. Cell 91:1055-1064.

Albrecht U, Zheng B, Larkin D, Sun ZS, Lee CC (2001) MPer1 and mper2 are essential for normal resetting of the circadian clock. J Biol Rhythms 16:100-104.

Arthur JSC, Cohen P (2000) MSK1 is required for CREB phosphorylation in response to mitogens in mouse embryonic stem cells. FEBS Lett 482:44-48.

Arthur JSC, Fong AL, Dwyer JM, Davare M, Reese E, Obrietan K, Impey S (2004) Mitogen- and stress-activated protein kinase 1 mediates cAMP response element-binding protein phosphorylation and activation by neurotrophins. J Neurosci 24:4324-4332.

Bae K, Weaver DR (2003) Light-induced phase shifts in mice lacking mPER1 or mPER2. J Biol Rhythms 18:123-133.

Barrie AP, Clohessy AM, Buensuceso CS, Rogers MV, Allen JM (1997) Pituitary adenylyl cyclase-activating peptide stimulates extracellular signalregulated kinase 1 or 2 (ERK 1/2) activity in a Ras-independent, mitogenactivated protein Kinase /ERK kinase 1 or 2-dependent manner in PC12 cells. J Biol Chem 272:19666-19671.

Bergström AL, Hannibal J, Hindersson P, Fahrenkrug J (2003) Lightinduced phase shift in the Syrian hamster (Mesocricetus auratus) is attenuated by the PACAP receptor antagonist PACAP6-38 or PACAP immunoneutralization. Eur J Neurosci 18:2552-2562.

Best JD, Maywood ES, Smith KL, Hastings MH (1999) Rapid resetting of the mammalian circadian clock. J Neurosci 19:828-835.

Butcher GQ, Dziema H, Collamore M, Burgoon PW, Obrietan K (2002) The p42/44 mitogen-activated protein kinase pathway couples photic input to circadian clock entrainment. J Biol Chem 277:29519-29525.

Butcher GQ, Lee B, Obrietan K (2003) Temporal regulation of lightinduced extracellular signal-regulated kinase activation in the suprachiasmatic nucleus. J Neurophysiol 90:3854-3863.

Butcher GQ, Lee B, Hsieh F, Obrietan K (2004) Light- and clock-dependent regulation of ribosomal S6 kinase activity in the suprachiasmatic nucleus. Eur J Neurosci 19:907-915.

Cermakian N, Pando MP, Thompson CL, Pinchak AB, Selby CP, Gutierrez L, Wells DE, Cahill GM, Sancar A, Sassone-Corsi P (2002) Light induction of a vertebrate clock gene involves signaling through blue-light receptors and MAP kinases. Curr Biol 12:844-848.
Chen D, Buchanan GF, Ding JM, Hannibal J, Gillette MU (1999) Pituitary adenylyl cyclase-activating peptide: a pivotal modulator of glutamatergic regulation of the suprachaismatic circadian clock. Proc Natl Acad Sci USA 96:13468-13473.

Cheng HY, Obrietan K, Cain SW, Lee BY, Agostino PV, Joza NA, Harrington ME, Ralph MR, Penninger JM (2004) Dexras1 potentiates photic and suppresses nonphotic responses of the circadian clock. Neuron 43:715-728.

Cobb MH (1999) MAP kinase pathways. Prog Biophys Mol Biol 71:479-500.

Colwell CS, Foster RG, Menaker M (1991) NMDA receptor antagonists block the effects of light on circadian behavior in the mouse. Brain Res 554:105-110.

Colwell CS, Michel S, Itri J, Rodriguez W, Tam J, Lelievre V, Hu Z, Waschek JA (2004) Selective deficits in the circadian light response in mice lacking PACAP. Am J Physiol Regul Integr Comp Physiol 287:R1194-R1201.

Coogan AN, Piggins HD (2003) Circadian and photic regulation of phosphorylation of ERK1/2 and Elk-1 in the suprachiasmatic nuclei of the Syrian hamster. J Neurosci 23:3085-3093.

Davies SP, Reddy H, Caivano M, Cohen P (2000) Specificity and mechanism of action of some commonly used protein kinase inhibitors. Biochem J 351:95-105.

Deak M, Clifton AD, Lucocq LM, Alessi DR (1998) Mitogen- and stressactivated protein kinase-1 (MSK1) is directly activated by MAPK and SAPK $2 / \mathrm{p} 38$, and may mediate activation of CREB. EMBO J 17:4426-4441.

Dziema H, Obrietan K (2002) PACAP potentiates L-type calcium channel conductance in suprachiasmatic nucleus neurons by activating the MAPK pathway. J Neurophysiol 88:1374-1386.

Dziema H, Oatis B, Butcher GQ, Yates R, Hoyt KR, Obrietan K (2003) The ERK/MAP kinase pathway couples light to immediate-early gene expression in the suprachiasmatic nucleus. Eur J Neurosci 17:1617-1627.

Ford SR, Leach FR (1998) Improvements in the application of firefly luciferase assays. Methods Mol Biol 102:3-20.

Frodin M, Jensen CJ, Merienne K, Gammeltoft S (2000) A phosphoserineregulated docking site in the protein kinase RSK2 that recruits and activates PDK1. EMBO J 19:2924-2934.

Fukada M, Gotoh I, Adachi M, Gotoh Y, Nishida E (1997) A novel regulatory mechanism in the mitogen-activated protein (MAP) kinase cascade. Role of nuclear export signal of MAP kinase kinase. J Biol Chem 272:32642-32648.

Gekakis N, Staknis D, Nguyen HB, Davis FC, Wilsbacher LD, King DP, Takahashi JS, Weitz CJ (1998) Role of the CLOCK protein in the mammalian circadian mechanism. Science 280:1564-1569.

Gonzalez GA, Montminy MR (1989) Cyclic AMP stimulates somatostatin gene transcription by phosphorylation of CREB at serine 133. Cell 59:675-680.

Gooley JJ, Lu J, Chou TC, Scammell TE, Saper CB (2001) Melanopsin in cells of origin of the retinohypothalmic tract. Nat Neurosci 4:1165.

Hannibal J, Ding JM, Chen D, Fahrenkrug J, Larsen PJ, Gillette MU, Mikkelsen JD (1997) Pituitary adenylate cyclase-activating peptide (PACAP) in the retinohypothalamic tract: a potential daytime regulator of the biological clock. J Neurosci 17:2637-2644.

Hannibal J, Moller M, Ottersen OP, Fahrenkrug J (2000) PACAP and glutamate are co-stored in the retinohypothalamic tract. J Comp Neurol 418:147-155.

Hannibal J, Vrang H, Card JP, Fahrenkrug J (2001) Light-dependent induction of cFos during subjective day and night in PACAP-containing ganglion cells of the retinohypothalamic tract. J Biol Rhythms 16:457-470.

Hannibal J, Hindersson P, Knudsen SM, Georg B, Fahrenkrug J (2002) The photopigment melanopsin is exclusively present in pituitary adenylate cyclase-activating polypeptide-containing retinal ganglion cells of the retinohypothalamic tract. J Neurosci 22:RC191(1-6).

Harrington ME, Hoque S, Hall A, Golombek D, Biello S (1999) Pituitary adenylate cyclase activating peptide phase shifts circadian rhythms in a manner similar to light. J Neurosci 19:6637-6642.

Hughes AT, Fahey B, Cutler DJ, Coogan AN, Piggins HD (2004) Aberrant gating of photic input to the suprachiasmatic circadian pacemaker of mice lacking the VPAC2 receptor. J Neurosci 24:3522-3526.

Jiang A, Craxton A, Kurosaki T, Clark EA (1998) Different protein tyrosine kinases are required for $\mathrm{B}$ cell antigen receptor-mediated activation of extracellular signal-regulated kinase, c-Jun NH2-terminal kinase 1, and p38 mitogen-activated protein kinase. J Exp Med 188:1297-1306.

Kalamatianos T, Kallo T, Piggins HD, Coen CW (2004) Expression of VIP 
and/or PACAP receptor mRNA in peptide synthesizing cells within the suprachiasmatic nucleus of the rat and in its efferent target sites. J Comp Neurol 475:19-35.

Kawaguchi C, Tanaka K, Isojima Y, Shintani N, Hashimoto S, Baba A, Nagai K (2003) Changes in light-induced phase shift of circadian rhythm in mice lacking PACAP. Biochem Biophys Res Commun 310:169-175.

Kopp MD, Meissl H, Dehghani F, Korf HW (2001) The pituitary adenylate cyclase-activating polypeptide modulates glutamatergic calcium signalling: investigations on rat suprachiasmatic nucleus neurons. J Neurochem 79:161-171.

Kornhauser JM, Nelson DE, Mayo KE, Takahashi JS (1990) Photic and circadian regulation of $\mathrm{c}$-fos gene expression in the hamster suprachiasmatic nucleus. Neuron 5:127-134.

McCoy CE, Campbell DG, Deak M, Bloomberg GB, Arthur JS (2005) MSK1 activity is controlled by multiple phosphorylation sites. Biochem J 387:507-517.

Meijer JH, Schwartz WJ (2003) In search of the pathways for light-induced pacemaker resetting in the suprachiasmatic nucleus. J Biol Rhythms 3:235-249.

Minami Y, Furuno K, Akiyama M, Moriya T, Shibata S (2002) Pituitary adenylate cyclase-activating polypeptide produces a phase shift associated with induction of mPer expression in the mouse suprachiasmatic nucleus. Neuroscience 113:37-45.

Moore RY (1996) Entrainment pathways and the functional organization of the circadian system. Prog Brain Res 111:103-119.

Moore RY, Silver R (1998) Suprachiasmatic nucleus organization. Chronobiol Int 15:475-487.

Nielsen HS, Hannibal J, Knudsen SM, Fahrenkrug J (2001) Pituitary adenylate cyclase-activating polypeptide induces period 1 and period 2 gene expression in the rat suprachiasmatic nucleus during late night. Neuroscience 103:433-441.

Obrietan K, Impey S, Storm DR (1998) Light and circadian rhythmicity regulate MAP kinase activation in the suprachiasmatic nuclei. Nat Neurosci 1:693-700.

Pearson G, Robinson F, Beers Gibson T, Xu BE, Karandikar M, Berman K, Cobb MH (2001) Mitogen-activated protein (MAP) kinase pathways: regulation and physiological functions. Endocr Rev 22:153-183.

Pierrat B, Correia JS, Mary JL, Tomas-Zuber M, Lesslauer W (1998) RSK-B, a novel ribosomal S6 kinase family member, is a CREB kinase under dominant control of p38alpha mitogen-activated protein kinase (p38alphaMAPK). J Biol Chem 273:29661-29671.

Pizzio GA, Hainich EC, Ferreyra GA, Coso OA, Golombek DA (2003) Circadian and photic regulation of ERK, JNK and p38 in the hamster SCN. NeuroReport 14:1417-1419.

Reppert SM, Weaver DR (2001) Molecular analysis of mammalian circadian rhythms. Annu Rev Physiol 63:647-676.

Robberecht P, Gourlet P, De Neef P, Woussen-Colle MC, Vandermeers-Piret MC, Vandermeers A, Christophe J (1992) Structural requirements for the occupancy of pituitary adenylate-cyclase-activating-peptide
(PACAP) receptors and adenylate cyclase activation in human neuroblastoma NB-OK-1 cell membranes. Discover of PACAP(6-38) as a potent antagonist. Eur J Biochem 207:239-246.

Roux PP, Blenis J (2004) ERK and p38 MAPK-activated protein kinases: a family of protein kinases with diverse biological functions. Microbiol Mol Biol Rev 68:320-344.

Rusak B, Robertson HA, Wisden W, Hunt SP (1990) Light pulses that shift rhythms induce gene expression in the suprachiasmatic nucleus. Science 248:1237-1240.

Shigeyoshi Y, Taguchi K, Yamamoto S, Takekida S, Yan L, Tei H, Moriya T, Shibata S, Loros J, Dunlap JC, Okamura H (1997) Light-induced resetting of mammalian circadian clock is associated with rapid induction of the mPer1 transcript. Cell 91:1043-1053.

Smith KJ, Carter PS, Bridges A, Horrocks P, Lewis C, Pettman G, Clarke A, Brown M, Hughes J, Wilkinson M, Bax B, Reith A (2004) The structure of MSK1 reveals a novel autoinhibitory conformation for a dual kinase protein. Structure 12:1067-1077.

Soloaga A, Thomson C, Wiggin GR, Rampersaund N, Dyson MH, Hazzalin CA, Mahadevan LC, Arthur JS (2003) MSK2 and MSK1 mediate the mitogen- and stress-induced phosphorylation of histone H3 and MHG14. EMBO J 22:2788-2797.

Travnickova-Bendova Z, Cermakian N, Reppert SM, Sassone-Corsi P (2002) Bimodal regulation of mPeriod promoters by CREB-dependent signaling and CLOCK/BMAL1 activity. Proc Natl Acad Sci USA 99:7728-7733.

Trischkau SA, Mitchell JW, Tyan SH, Buchanan GF, Gillette MU (2003) $\mathrm{Ca}^{2+} / \mathrm{cAMP}$ response element-binding protein (CREB)-dependent activation of Perl is required for light-induced signaling in the suprachiasmatic nucleus circadian clock. J Biol Chem 278:718-723.

Uz T, Manev H (1998) Circadian expression of pineal 5-lipoxygenase mRNA. NeuroReport 9:783-786.

Wakamatsu H, Takahashi S, Moriya T, Inouye ST, Okamura H, Akiyama M, Shibata S (2001) Additive effect of mPerl and mPer2 antisense oligonucleotides on light-induced phase shift. NeuroReport 12:127-131.

Wang XS, Diener K, Manthey CL, Wang S, Rosenzweig B, Bray J, Delaney J, Cole CN, Chan-Hui PY, Mantlo N, Lichenstein HS, Zukowski M, Yao Z (1997) Molecular cloning and characterization of a novel p38 mitogenactivated protein kinase. J Biol Chem 272:23668-23674.

Wiggin GR, Soloaga A, Foster JM, Murray-Tait V, Cohen P, Arthur JS (2002) MSK1 and MSK2 are required for the mitogen- and stress-induced phosphorylation of CREB and ATF1 in fibroblasts. Mol Cell Biol 22:2871-2881.

Williams MR, Arthur JS, Balendran A, van der Kaay J, Poli V, Cohen P, Alessi DR (2000) The role of 3-phosphoinositide-dependent protein kinase 1 in activating AGC kinases defined in embryonic stem cells. Curr Biol 10:439-448.

Yamaguchi S, Mitsui S, Miyake S, Yan L, Onishi H, Yagita K, Suzuki M, Shibata S, Kobayashi M, Okamura H (2000) The 5' upstream region of $\mathrm{mPer} 1$ gene contains two promoters and is responsible for circadian oscillation. Curr Biol 10:873-876. 